\title{
The Prevalence, Etiologic Agents and Risk Factors for Urinary Tract Infection Among Spinal Cord Injury Patients
}

\author{
Turhan Togan ${ }^{1, *}$; Ozlem Kurt Azap ${ }^{1}$; Elif Durukan ${ }^{2}$; Hande Arslan $^{1}$ \\ ${ }^{1}$ Department of Infectious Diseases and Clinical Microbiology, Faculty of Medicine, Baskent University, Ankara, Turkey \\ ${ }^{2}$ Department of Public Health, Baskent University Faculty of Medicine, Ankara, Turkey \\ *Corresponding author: Turhan Togan, Application and Research Center, Hocacihan Mah. Saray Cad. No.1 Selcuklu Konya, Ankara, Turkey. Tel: +90-3322570606-3113, Fax: +90- \\ 3322570637, E-mail: drtogant@hotmail.com
}

Received: October 31, 2012; Revised: January 23, 2013; Accepted: February 19, 2013

\begin{abstract}
Background: Urinary tract infections (UTIs) are important causes of morbidity and mortality in patients with spinal cord injury and 22\% of patients with acute spinal cord injury develop UTI during the first 50 days.

Objectives: The aim of this study was to determine the prevalence, etiologic agents and risk factors for asymptomatic bacteriuria and symptomatic urinary tract infections in patients with spinal cord injury.

Patients and Methods: This was a prospective investigation of spinal cord injury patients with asymptomatic bacteriuria and symptomatic urinary tract infections in Baskent University Medical Faculty Ayas Rehabilitation Center and Ankara Physical Therapy and Rehabilitation Center between January 2008 and December 2010. The demographic status, clinical and laboratory findings of 93 patients with spinal cord injury were analyzed in order to determine the risk factors for asymptomatic or symptomatic bacteriuria

Results: Sixty three (67.7\%) of 93 patients had asymptomatic bacteriuria and 21 (22.6\%) had symptomatic urinary tract infection. Assessment of the frequency of urinary bladder emptying methods revealed that 57 (61.3\%) of 93 patients employed permanent catheters and 24 (25.8\%) employed clean intermittent catheterization. One hundred and thirty-five (48.0\%) of 281 strains isolated form asymptomatic bacteriuria attacks and 16(66.6\%) of 24 strains isolated from symptomatic urinary tract infection attacks, totaling 151 strains, had multidrug resistance $(\mathrm{P}>0.05)$. One hundred (70.4\%) of 142 Escherichia coli strains and 19 (34.5\%) of 55 Klebsiella spp strains proliferated in patients with asymptomatic bacteriuria; 8 (80\%) of 10 E. coli strains and 4 (80\%) of 5 Klebsiella spp. strains were multidrug resistant.

Conclusions: The most common infectious episode among spinal cord injury patients was found to be urinary tract infection. E. coli was the most common microorganism isolated from urine samples. Antibiotic use in the previous 2 weeks or 3 months, hospitalization during the last one-year and previous diagnosis of urinary tract infection were the risk factors identified for the development of infections with multi-drug resistant isolates. Urinary catheterization was found to be the only independent risk factor contributing to symptomatic urinary tract infection.
\end{abstract}

Keywords: Urinary Tract Infections; Spinal Cord Injury; Asymptomatic Bacteriuria; Symptomatic Bacteriuria

\section{Background}

Urinary tract infections (UTIs) are important causes of morbidity and mortality in patients with spinal cord injury (SCI). It has been reported that $22 \%$ of patients with acute SCI develop UTI during the first 50 days and annual UTI incidence in patients with chronic SCI is nearly $20 \%$ (16 ). Mortality rate in individuals with spinal cord injury is as high as $6.3 \%$ in the first year after the injury, whereas it significantly decreases in the subsequent years (7).

Cause of mortality in individuals with SCI is mainly respiratory diseases with a rate of $21.7 \%$ while heart diseases rank second with a rate of $12.6 \%$ and infections rank third with a rate of $9.4 \%$ (8). While urinary system infections are major causes of mortality and morbidity in patients with SCI, mortality due to urinary sepsis has decreased to $10-15 \%$ with improved management (2).

\section{Objectives}

The aim of this study was to determine the prevalence and causative agents of asymptomatic bacteriuria (ASB) and symptomatic urinary system infection (SUSI), and risk factors for UTI in patients with SCI.

\section{Patients and Methods}

A total of 93 patients with SCI were followed at Baskent University Medical Faculty Ayas Rehabilitation Center and Ankara Physical Therapy and Rehabilitation Center between $15^{\text {th }}$ of February 2008 and $30^{\text {th }}$ of June 2009. Patients belonged to the age group of 18-65 years. Patients with SCI were prospectively where followed Subjects were visited every week. A form was filled for each patient; containing demographic features, clinical signs and symp-

Implication for health policy/practice/research/medical education:

Urinary catheterization was found to be the only independent risk factor contributing to symptomatic urinary tract infection in patients with spinal cord injury.

Copyright (C) 2014,Ahvaz Jundishapur University of Medical Sciences; Published by Kowsar Corp. This is an open-access article distributed under the terms of the Creative Commons Attribution License, which permits unrestricted use, distribution, and reproduction in any medium, provided the original work is properly cited. 
toms, laboratory and imaging findings, isolated microorganisms and their sensitivities to antibiotics. Urinary samples and culture was taken from patients during each visit. Antibiotic sensitivities were determined by the Kirby-Bauer disk diffusion method in compliance with Clinical Laboratory Standards Institute (CLSI) rules (9).

The threshold level for significant bacteriuria was accepted as $10^{5} \mathrm{cfu} / \mathrm{mL}$ (10). A patient was considered to have symptomatic urinary system infection if he/she had significant bacteriuria plus one of the following clinical findings; body temperature $>38^{\circ} \mathrm{C}$, abdominal pain, reflex perspiration, urinary incontinence, increased spasticity, suprapubic pain, flank pain, frequent urination, dysuria, urgency, urinary incontinence, foul smell in urine, and cloudy urine (11-14). Patients with significant bacteriuria but no fever or clinical complaints were considered to have asymptomatic bacteriuria $(5,15)$. Patients with bacteriuria and fever but no urinary complaint, whose fever was due to another cause, were considered to have "non-UTI infection".

All patients were followed up with weekly complete blood counts and CRP tests. Eighty-four patients underwent abdominal ultrasonography to screen for trabeculations in urinary bladder (present/absent), parenchymal changes in kidney (present/absent), and vesicoureteral reflux (present/absent). Strains resistant to at least three of quinolones, beta-lactams, aminoglycosides, and co-trimoxazole group antibiotics were defined as "multi-drug resistant (MDR) strains".

\subsection{Statistical Analysis}

Study data were analyzed using Statistical Package for the Social Sciences (SPSS) 17.0 (SPPS Inc., Chicago, IL, USA) software. Categorical variables were compared by the ChiSquare and Fisher's exact Chi-Square test; comparison of differences of continuous variables was carried out with $t$ test in independent groups. Risk factors for bacteriuria were determined with logistic regression analysis. A patient value of less than 0.05 was considered significant.

\section{Results}

Ninety-three patients ( 78 males, 15 females) were included. Mean age was $35.65 \pm 13.11$. Spinal cord injury level was cervical in $30.5 \%$, thoracic in $63 \%$, lumbar in $6.5 \%$. Seventythree (78.5\%) patients were paraplegic and 17 (18.3\%) were quadriplegic. Three (3.2\%) patients had minor spinal cord injury with associated paresthesia. The most common causes of spinal cord injury were traffic accidents in $44.1 \%$ and falls in $38.7 \%$. Firearm wounds, trauma, vertebral mass, electric shock, and operation were other causes, in descending order. Mean duration of urinary catheterization was 2.5 months. A mean of $4.2 \pm 1.2$ visits, each oneweek apart, took place. A total of 397 visits were paid to 93 patients. Bacteriuria was detected in 84 (90.3\%) of 93 patients, while $9(9.7 \%)$ had no proliferation. Sixty three (67.7\%) of 93 patients had ASB and 21 (22.6\%) had SUSI. Twenty-one patients with the diagnosis of SUSI developed
24 attacks and 63 patients with ASB developed 281 attacks, totaling 305 bacteriuria attacks. Twenty-seven of 93 patients developed 30 infectious attacks during the followup. Twenty-one patients had a total of 24 SUSI attacks and 6 patients had infections of other systems (4 respiratory system infections, 1 bacteremia and 1 dental abscess).

Assessment of the frequency of urinary bladder emptying methods revealed that 57 (61.3\%) of 93 patients employed permanent catheters and 24 (25.8\%) employed clean intermittent catheterization (CIC). Twelve (12.9\%) patients were able to urinate spontaneously. ASB/SUSI development was assessed and it was found that 34 (59.6\%) of 57 patients with permanent catheters developed ASB, $17(29.8 \%)$ had SUSI, whereas $6(10.6 \%)$ had no proliferation.

Twenty (83.3\%) of 24 patients in whom clean intermittent catheterization was used, were diagnosed with ASB and 4 (16.7\%) were diagnosed with SUSI. Three of 12 patients urinating spontaneously had no proliferation. There was no significant relationship between catheterization type and ASB or SUSI development.

Bladder emptying method in 63 followed patients with asymptomatic bacteriuria was with a permanent catheter in 34 (53.9\%), CIC in 20 (31.7\%), and spontaneous urination in 9 (14.4\%). Seventeen (80.9\%) of 21 patients followed up with symptomatic UTI had permanent catheters and 4 (19.1\%) had CIC. No patients among those with SUSI urinated spontaneously. Six of 9 patients with no proliferation had permanent catheterization while 3 were able to urinate spontaneously. There was no significant difference between bladder emptying methods in terms of bacteriuria.

Agents isolated from ASB and SUSI attacks are mentioned in Table 1.

Table 1. Distribution of Agents Isolated from Asymptomatic Bacteriuria and Symptomatic UTI Attacks

\begin{tabular}{|c|c|c|}
\hline Microorganism & ASB, No. (\%) & SUSI, No. (\%) \\
\hline E. coli & $142(50.5)$ & $10(41.7)$ \\
\hline Klebsiella spp. & $55(19.6)$ & $5(20.8)$ \\
\hline Enterococcusspp. & $23(8.2)$ & $2(8.3)$ \\
\hline Pseudomonasspp. & $15(5.3)$ & $2(8.3)$ \\
\hline KNS $^{\mathrm{a}}$ & $8(2.9)$ & - \\
\hline Acinetobacterspp. & $7(2.5)$ & $3(12.6)$ \\
\hline Proteus spp. & $6(2.1)$ & - \\
\hline S.aureus & $4(1.4)$ & - \\
\hline Candidaspp. & $4(1.4)$ & $2(8.3)$ \\
\hline Enterobacterspp. & $2(0.8)$ & - \\
\hline other $^{b}$ & $15(4.6)$ & - \\
\hline Total & $281(100.0)$ & $24(100.0)$ \\
\hline
\end{tabular}


There was no statistical difference between strains isolated from patients diagnosed with symptomatic UTI and ASB in terms of antibiotic resistance (Tables 2 and 3). Thirty (47.6\%) from 63 patients were followed up for ASB, 11 (52.4\%) of 21 patients were followed up for SUSI, and 4
(44.4\%) of 9 patients with no proliferation had a history of antibiotic use in the last 3 months. Although antibiotic use was more prevalent in those with bacteriuria, there was no significant difference between the groups ( $\mathrm{P}>$ $0.05)$.

Table 2. Rates of Antibiotic Resistance of E.coli Strains Isolated From Symptomatic UTI and ASB Attacks.

\begin{tabular}{|c|c|c|c|}
\hline Antibiotic & ASB, No. $(\%)^{\mathrm{a}}$ & SUSI, No. (\%) ${ }^{\mathrm{a}}$ & PValue $^{\mathbf{b}}$ \\
\hline Ampicillin & $124(87.3)$ & $8(80.0)$ & $>0.05$ \\
\hline Cefuroxime & $101(71.1)$ & $7(70.0)$ & $>0.05$ \\
\hline Co-Trimoxazole & $95(66.9)$ & $8(80.0)$ & $>0.05$ \\
\hline Ciprofloxacin & $87(61.3)$ & $6(60.0)$ & $>0.05$ \\
\hline Amoxicillin & $85(59.9)$ & $3(30.0)$ & $>0.05$ \\
\hline Ceftriaxone & $71(50.0)$ & $5(50.0)$ & $>0.05$ \\
\hline Gentamicin & $57(40.1)$ & $2(20.0)$ & $>0.05$ \\
\hline Cefepime & $29(20.4)$ & 0 & $>0.05$ \\
\hline Piperacillin-Tazobactam & $12(8.5)$ & 0 & $>0.05$ \\
\hline Amikacin & $7(4.9)$ & 0 & $>0.05$ \\
\hline Imipenem & 0 & 0 & 0 \\
\hline Meropenem & 0 & 0 & 0 \\
\hline
\end{tabular}

a The percentage of resistant strains

b Ki-Kare test

Table 3. Rates of Antibiotic Resistance of Klebsiella spp. Strains Isolated From Patients With Symptomatic UTI and ASB

\begin{tabular}{llll}
\hline Antibiotic & ASB No. $(\%)^{\mathrm{a}}$ & SUSI, No. $(\%)^{\mathrm{a}}$ & PValue $^{\mathbf{b}}$ \\
\hline Co-Trimoxazole & $23(41.8)$ & $4(80.0)$ & $>0.05$ \\
Cefuroxime & $22(40.0)$ & $3(60.0)$ & $>0.05$ \\
Amoxicillin & $21(38.2)$ & $5(100.0)$ & $>0.05$ \\
Ceftriaxone & $19(34.5)$ & $1(20.0)$ & $>0.05$ \\
\hline Ciprofloxacin & $16(29.1)$ & $3(60.0)$ & $>0.05$ \\
Gentamicin & $12(21.8)$ & $2(40.0)$ & $>0.05$ \\
Cefepime & $12(21.8)$ & $1(20.0)$ & $>0.05$ \\
Piperacillin-Tazobactam & $4(7.3)$ & $2(40.0)$ & $>0.05$ \\
Amikacin & $3(5.5)$ & $1(20.0)$ & $>0.05$ \\
\hline Imipenem & 0 & 0 & \\
\hline Meropenem & 0 & 0 & \\
\hline
\end{tabular}

a The percentage of resistant strains

b Ki-kare test

One hundred and thirty-five (48.0\%) of 281 strains isolated form ASB attacks and 16 (66.6\%) of 24 strains isolated from SUSI attacks, totaling 151 strains, had multidrug resistance $(P>0.05)$. One hundred $(70.4 \%)$ of $142 \mathrm{E}$. coli strains and 19 (34.5\%) of 55 Klebsiella. spp strains proliferated in patients with asymptomatic bacteriuria; 8 (80\%) of 10 E.coli strains and 4 (80\%) of 5 Klebsiella spp. strains were multidrug resistant. The ratio of strains, which were multidrug resistant, was significantly higher in patients diagnosed with SUSI compared to those diagnosed with
$\operatorname{ASB}(P<0.05)$

Nineteen $(55.8 \%)$ of 34 patients from whom multidrug resistant bacteria were isolated had a history of antibiotic use in the last 3 months, 14 (47.1\%) had a history of hospitalization within the last year, and $13(38.2 \%)$ had a history of UTI. Leukocytosis was detected in 30 (14.1\%) of 281 ASB attacks and 8 (38.1\%) of 24 SUSI attacks. The likelihood of SUSI in patients with leukocytosis was 3.95 times greater $(\mathrm{OR}=3.95 ; 95 \% \mathrm{CI}=1.49-10.51)$. CRP elevation was detected in $88(57.9 \%)$ of 281 ASB attacks, whereas 11 (84.6\%) of 24 
SUSI attacks had CRP elevation $(P>0.05)$. Pyuria was present in 92 (44\%) of 281 patients with ASB attack, 11 (55\%) of 24 patients with SUSI. $(\mathrm{P}<0.05)$. Nitrite positivity in urine was present in 131 (62.4\%) of 281 ASB attacks and 14 (70\%) of 24 SUSI attacks $(\mathrm{P}>0.05)$.

Eighty-four patients underwent urinary ultrasonography and 77 (91.7\%) had normal results whereas 7 (8.3\%) had urinary system pathologies (nephrolithiasis, vesicoureteral reflux, benign prostate hypertrophy, nephrectomy). Three (3.6\%) patients had urinary stones. Forty-one (48.8\%) from a total of 84 patients with bacteriuria and $4(44.4 \%)$ of 9 patients without bacteriuria had a history of hospitalization within the previous year. Twenty-seven (42.8\%) of 63 followed patients with ASB and 10 (47.6\%) of 21 followed patients with SUSI had a history of hospitalization within the previous year. Those with bacteriuria had a greater rate of hospitalization; albeit statistically insignificant $(\mathrm{P}>0.05)$.

Thirty-seven (44\%) of 84 patients had a history of SUSI within the previous year. 4 (44\%) of 9 patients with no bacteriuria had a history of SUSI within the previous year. Forty-six (54.7\%) of 84 patients had a history of antibiotic use in the last 3 months while 4 (44.4\%) of those 9 without bacteriuria had a similar history. The most commonly used antibiotic class was quinolones (Table 4).

\begin{tabular}{ll}
\hline \multicolumn{2}{l}{ Table 4. Antibiotics Used in the Last 3 Months } \\
\hline Antibiotic & Patient, No. (\%) \\
\hline Quinolone & $15(32.6)$ \\
Co-Trimoxazole & $11(23.9)$ \\
Cephalosporin & $7(15.2)$ \\
Penicillin & $6(13.0)$ \\
Carbapenem & $3(6.5)$ \\
Other $^{\mathrm{a}}$ & $4(8.7)$ \\
\hline Total & $46(100.0)$ \\
\hline
\end{tabular}

${ }^{\mathrm{a}}$ Fluconazole, Nitrofurantoin

The mean interval from admission to SUSI attack was $22.20 \pm 15,73$ days. There was no significant difference between spinal cord injury levels in terms of having SUSI $(P>0.05)$.There was no significant difference between genders in terms of having SUSI $(\mathrm{P}>0.05)$. SUSI was not related to gender, age, duration or level of injury, duration of catheterization, having UTI or hospitalization within the previous year, antibiotic use within the last 3 months, presence of decubitus ulcers, presence of stones, increased CRP levels, presence of pyuria, nitrite positivity, presence of trabeculations, or renal parenchymal changes observed at USG.

\section{Discussion}

A total of 93 patients, diagnosed with SCI and treated at physical therapy and rehabilitation units, were included in this study. Percentage of males was 83.9, with a male- to female ratio similar to world data. In United States of America, approximately 18-33\% of patients with SCI were female and $67-82 \%$ were male $(16,17)$. Mean age for SCI has been reported as $37 \pm 11.5$ years worldwide $(18,19)$ and 35.9 years in the United States (8). Turkish studies have reported a mean age of 35.5 years (20). Mean age of our patients was $35.65 \pm 13.11$ years, which was similar to the world average.

Eighty-four (90.3\%) of 93 patients in our study had bacteriuria whereas 9 (9.7\%) had no proliferation. Ruz et al. reported that the incidence of bacteriuria was 2.72 attacks/100 patient days patient hospitalization days and for UTI this was 0.68 attack/100 patient days patient hospitalization days in patients for whom a urinary drainage method was employed (21). Twenty-five percent of 84 patients with bacteriuria had a SUSI attack and 75\% had an ASB attack. Fifty-seven (61.3\%) of 93 patients used a permanent catheter, 24 (25.8\%) used a CIC. Twelve (12.9\%) patients were able to urinate spontaneously. Six (66.6\%) of $9(9.7 \%)$ patients with no proliferation were followed with a permanent catheter, 3 (33.4\%) of them were able to urinate spontaneously. These patients did not experience significant bacteriuria during their hospital stay despite prolonged catheterization.

Oz et al. in a study including 63 patients with SCI found that the rate of bacteriuria was 53.3\% in patients using CIC and $82.9 \%$ in those with permanent catheters (22). Ruz et al. in a study examining 128 patients with SCI, reported that the rate of bacteriuria was 5 attacks/100 patient days patient hospitalization days, 2.95 attacks/100 patient days patient hospitalization days, 2.41 attacks/100 patient days patient hospitalization days, and 0.96 attack/100 patient days patient hospitalization days in permanent catheterization, clean intermittent catheterization, and condom catheter in males and supra pubic catheterization in females, respectively (21). The same study reported a bacteriuria rate of 0.33 attack/100 patient days patient hospitalization days in incomplete injuries with normal function of urination (21). The highest bacteriuria rate was due to permanent catheterization while the lowest rate was observed in those who were able to urinate. The finding of a higher bacteriuria rate in permanent catheterization compared to CIC is in agreement with the literature.

In a study from Portugal, $24.6 \%$ of catheterized patients were detected to have SUSI. It was also observed that UTI was more frequent in patients with a permanent catheter compared to those employing CIC (21). Oz et al. reported a SUSI rate of $61.5 \%$ in those with a urinary catheter (22). The highest UTI rate was observed in those with a permanent catheter. Our finding of a higher SUSI attack rate in permanent catheter users compared to CIC users is consistent with the literature. Microorganisms commonly isolated from urine cultures of patients with SCI are E.coli, Pseudomonas spp., Klebsiella spp., Proteus spp., Serratia spp., Providencia spp., Enterococci, and Staphylococci (23-25). Inspection of 305 agents isolated from a total 
of 84 patients followed up for bacteriuria revealed that the most common agent was E.coli with a percentage of $49.9 \%$, followed by (in descending order) Klebsiella spp. (19.7\%), Enterococcus spp. (8.2\%), and Pseudomonas spp. (5.6\%).

In our study, agents responsible for SUSI in SCI patients were E. coli in $41.7 \%$, Klebsiella spp. in $20.8 \%$, and Acinetobacter spp. in $12.6 \%$. E. coli (50.5\%) was the most commonly isolated pathogen in patients with ASB. As one can observe, E. coli takes the top place in agent distribution in patients followed up with SUSI and ASB. There was no significant difference between the distribution of agents isolated from patients with asymptomatic bacteriuria and patients with symptomatic urinary system infection. Yadav et al. reported that K. pneumonia and P. aeruginosa proliferated in urine cultures in $75 \%$ of patients when CIC was administered during early stages of SCI. In addition they reported that the most commonly isolated organism was E. coli in cases with a prolonged follow-up (26). Polymicrobial infection comes into question with prolonged catheterization, which may give rise to proliferation of rare agents such as Morganella spp. and Providencia spp.(27). Despite the excellent care of patients catheterized for a long time, bacteriuria is inevitable. Both new bacteriuria episodes and persistent bacteriuria caused by some bacteria species may last for weeks or even months $(12,28)$.

In spinal cord injury, UTI agents are generally polymicrobial (1). In a study by Dedeic-Ljubovi et al. UTI attacks were generally polymicrobial while $44 \%$ had single bacteria species isolated (29). Since the significant proliferation threshold in our study was assumed as $10^{5} \mathrm{cfu} / \mathrm{mL}$, we did not observe a polymicrobial bacteriuria attack (10). Some studies consider $10^{5} \mathrm{cfu} / \mathrm{mL}$ as the threshold value for bacteriuria while many studies consider proliferations at $10^{2} \mathrm{cfu} / \mathrm{mL}$ significant since such proliferations increase in subsequent days (30). Since proliferations at the level of $10^{2} \mathrm{cfu} / \mathrm{mL}$ have been considered abnormal, rates of polymicrobial bacteriuria in our study were inconsistent with the literature.

We studied antibiotic sensitivities of isolated agents to guide empiric antimicrobial treatment of SUSI (31). Among isolated strains, $61.2 \%$ of E. coli, and $31.7 \%$ of Klebsiella spp. strains were resistant to ciprofloxacin, $67.8 \%$ of E. coli and $45.0 \%$ of Klebsiella spp. were resistant toco-trimoxazole, $50.0 \%$ of E. coli and 33.3\% of Klebsiella spp. were resistant to ceftriaxone, and $38.8 \%$ of E. coli and $23.3 \%$ of Klebsiella spp. were resistant to gentamicin. All isolated strains were sensitive to carbapenems. According to our results we defined that the efficiency of ciprofloxacin has been decreased but carbapenems has been effective in treatment of urinary system infections.

As studies of patients with spinal cord injury have been sparse, data on antimicrobial sensitivity are also quite limited. In a thesis study from Trakya University Medical Faculty in 1995, all 10 E. coli strains isolated from patients with urinary catheters were sensitive to amikacin, car- bapenems, and quinolones, where as $70 \%$ were resistant to co-trimoxazole (32). One study from Portugal reported that quinolone resistance has been increasing in bacteria isolated from catheter-related UTI attacks and majority of strains were more sensitive to amoxicillin than quinolones (33). Our results, along with other studies, show that sensitivity to quinolone and co-trimoxazole has been decreasing.

It has been reported that $2-4 \%$ of patients with spinal cord injury who are urinary catheterized have bacteremia $(34,35)$. Bacteremia attacks in our study were independent of SUSI. Falknier (36) reported that bacteremia prevalence was high in patients with prolonged catheterization. Our data on bacteremia were not consistent with the previous literature. This is possibly secondary to the small sample size and lack of blood cultures taken at febrile periods. Difficult-to-cure multidrug-resistant bacteria also complicate UTI therapy. In a study by DedeicLjubovi et al. 55.3\% of 3963 strains, isolated from patients with spinal cord injury who were catheterized, were multidrug-resistant. $87.8 \%$ of $A$. baumannii strains, $86.7 \%$ of $P$. rettgeri strains, $85.4 \%$ of $P$. aeruginosa strains, $84.3 \%$ of P. stuarti strains, and $81 \%$ of M. morganii strains were multidrug-resistant (29).

There were no significant difference between two groups (135 (48.0\%) of 281 strains isolated from ASB attacks and 16 (66.6\%) of 24 strains isolated from SUSI attacks, $(\mathrm{P}>0.05)$. We defined multidrug resistance as resistance to at least three of the quinolones, beta-lactams, and aminoglycosides groups and obtained a high rate of multidrug-resistance, consistent with the literature. Among patients from whom multidrug-resistant bacteria were isolated, $44.1 \%$ had a history of using antibiotics in the previous 2 weeks, $55.8 \%$ had used antibiotics within the last 3 months, $47.1 \%$ were hospitalized in the previous year, and $38.2 \%$ had had UTI within the last year. These rates were not statistically significant but recent antibiotic use, history of hospitalization or UTI within the previous 1 year should suggest that an infection has developed by multidrug-resistant bacteria. This is because nearly half of the patients from whom multidrug-resistant bacteria were isolated had these factors, albeit statistically non-significant.

In 91 of 93 patients in our study, body temperature was $37.5^{\circ} \mathrm{C}$ or below. Two of the patients followed-up for symptomatic UTI had a body temperature of $38-40^{\circ} \mathrm{C}$, which dropped with treatment. Therefore, there was no significant relationship between SUSI attack and body temperature. Mean SCI level was T6 or higher. This condition may suggest a tendency towards hypothermia due to the possible alteration of thermoregulatory system response. Studies have shown that $32-40 \%$ of patients with SCI had fever during their UTI attack $(37,38)$. In the study by Oz et al. CRP was high in $44 \%$ of SCI patients with UTI (22). In our study, on the other hand, CRP elevation was present in $57.9 \%$ of ASB attacks and $84.6 \%$ of SUSI attacks. Both studies have not found any significant relationship 
between increased CRP level and UTI development.

While Oz et al. detected leukocytosis in 19\% of UTI cases (22), we found leukocytosis in $14.1 \%$ of ASB attacks and $38.1 \%$ of SUSI attacks. Likelihood of SUSI in patients with leukocytosis was 3.95 times greater. Pyuria in our study was present in $44 \%$ of patients with ASB and $55 \%$ of those with SUSI. Presence of SUSI was not a significant predictor of SUSI. One study demonstrated that pyuria was associated with bacteriuria with a sensitivity of $74 \%$ and specificity of $95.9 \%$ (39). However, presence of pyuria is not a sufficient criterion for the diagnosis of bacteriuria. Furthermore, it does not distinguish symptomatic versus asymptomatic urinary system infection since other inflammatory conditions of genitourinary system may cause pyuria (5). Deresinski and Perkash, in a study of 70 patients with SCI, reported that bacteriuria was accompanied by pyuria in $97.4 \%$ of patients, whereas $40.6 \%$ of patients were asymptomatic despite significant pyuria (39). $\mathrm{Oz}$, unlike these results, detected pyuira as the sole indicator for the development of UTI in patients with SCI (22).

Abdominal ultrasonographic examinations of patients with SCI, have indicated that urinary bladder pathologies were more common in males and in those with a higher level of injury, longer disease duration, and complete injury. Bladder stones and bladder trabeculations were reported in $2 \%$ and $1.8 \%$ of patients with SCI, respectively (40). Oz et al. detected renal parenchymal changes on urinary USG examination in $9.5 \%$ of patients. Investigation of the presence of stones and renal parenchymal changes revealed that 10 patients out of 46 had bladder trabeculations and 4 had renal parenchymal changes (22). Eightyfour patients in our study underwent urinary ultrasonographic examination and 3 (3.6\%) had nephrolithiasis. This ratio was consistent with the literature.

Ruz et al. defined risk factors for symptomatic UTI development in patients with spinal cord injury. They concluded that the following were risk factors for symptomatic UTI: level of cervical lesion, invasive interventions, and a urinary catheterization lasting longer than 30 days (21). We periodically monitored 93 patients for 18 months and a total of 397 visits were performed. The single independent risk factor for development of symptomatic UTI was catheterization. We found that $47.6 \%$ of patients with UTI had a history of antibiotic use within the last 2 weeks and $52.4 \%$ within the last 3 months, $47.6 \%$ had been hospitalized within the last year and $38.1 \%$ had had UTI within the last year. Many studies have defined these findings as risk factors. We think that we could not identify them as independent risk factors because of our limited sample size.

\section{Acknowledgements}

There are no Acknowledgments.

\section{Authors' Contribution}

Study group: Turhan Togan, Ozlem Kurt| Azap Hande Arslan, Statistical analysis: Elif Durukan

\section{Financial Disclosure}

The authors declare no financial disclosure.

\section{Funding/Support}

There is no Funding/Support.

\section{References}

1. Biering-Srensen Fin, Bagi Per, Hoiby Niels. Urinary Tract Infections in Patients with Spinal Cord Lesions. Drugs. 2001;61(9):12751287.

2. Garcia Leoni ME, Esclarin De Ruz A. Management of urinary tract infection in patients with spinal cord injuries. Clin Microbiol In fec. 2003;9(8):780-785.

3. Montgomerie JZ. Infections in patients with spinal cord injuries. Clin Infect Dis. 1997;25(6):1285-90.

4. Nicolle Lindsay E. Asymptomatic bacteriuria. Infect Dis Clin North Am. 2003;17(2):367-394.

5. Nicolle LE, Bradley S, Colgan R, Rice JC, Schaeffer A, Hooton TM, et al. Infectious Diseases Society of America guidelines for the diagnosis and treatment of asymptomatic bacteriuria in adults. Clin Infect Dis. 2005;40(5):643-54.

6. Siroky Mike B. Pathogenesis of bacteriuria and infection in the spinal cord injured patient. Am J Med. 2002;113(1):67-79.

7. DeVivo Michael J, Stover Samuel L, Black Karin J. Prognostic factors for 12-year survival after spinal cord injury. Arch Physical Med Rehabilit. 1992;73(2):156-162.

8. Bryce TN, Ragnarsson KT. Pain after spinal cord injury. Phys Med Rehabilitation Clin North Am. 2000;11(1):157.

9. Wayne PA. Performance standards for antimicrobial susceptibility testing. Ninth informational supplement NCCLS document M100S9. National Committee for Clinical Laboratory Standards. 2008

10. Wilson ML, Gaido L. Laboratory diagnosis of urinary tract infections in adult patients. Clin Infect Dis. 2004;38(8):1150-8.

11. Cardenas DD, Mayo ME. Management of bladder dysfunction. Braddom RL editor. Philadelphia: WB Saunders Company; 2007.

12. Sedor John, Mulholland SGrant. Hospital-Acquired Urinary Tract Infections Associated with the Indwelling Catheter. Urologic Clinics of North America. 1999;26(4):821-828.

13. Stamm WE. Urinary tract infections. 4 ed. Bennett JV Brachman PS ed editor. Philadelphia: Lipincott-Raven Publishers; 1998.

14. Sugarman B. Fever in recently injured quadriplegic persons. Arch Phys Med Rehabil.1982;63(12):639-40.

15. Colgan Richard, Nicolle Lindsay E, Mcglone Andrew, Hooton Thomas M. Asymptomatic bacteriuria in adults. Women. 2006;3:15.0.

16. Cardenas DD, Hoffman JM, Kirshblum S, McKinley W. Etiology and incidence of rehospitalization after traumatic spinal cord injury: a multicenter analysis. Arch Phys Med Rehabil. 2004;85(11):1757-63.

17. Paker N, Soy D, Kesiktas N, Nur Bardak A, Erbil M, Ersoy S, et al Reasons for rehospitalization in patients with spinal cord injury: 5 years' experience. Int J Rehabil Res. 2006;29(1):71-6.

18. Fuhrer MJ, Rintala DH, Hart KA, Clearman R, Young ME. Relationship of life satisfaction to impairment, disability, and handicap among persons with spinal cord injury living in the community. Arch Phys Med Rehabil. 1992;73(6):552-7.

19. Lin KH, Chuang CC, Kao MJ, Lien IN, Tsauo JY. Quality of life of spinal cord injured patients in Taiwan: a subgroup study. Spinal Cord. 1997;35(12):841-9.

20. Karacan I, Koyuncu H, Pekel O, Sumbuloglu G, Kirnap M, Dursun $\mathrm{H}$, et al. Traumatic spinal cord injuries in Turkey: a nation-wide epidemiological study. Spinal Cord. 2000;38(11):697-701.

21. De Ruz AE, Leoni EG, Cabrera RH. Epidemiology and risk factors for urinary tract infection in patients with spinal cord injury. $J$ Urol. 2000;164(4):1285-1289.

22. Öz Bengi, Şengül İlker, Ölmez Neșe, Memiș Asuman. [Associated Factors with Urinary Tract Infection in Spinal Cord Injured Patients with Applied Bladder Management]. TurkJ Phys Med Rehab. 2009;55:55-59. 
23. Cardenas Diana D, Hooton Thomas M. Urinary tract infection in persons with spinal cord injury. Arch Phys Med Rehab. 1995;76(3):272-280.

24. Waites KB, Canupp KC, DeVivo MJ. Eradication of urinary tract infection following spinal cord injury. Paraplegia. 1993;31(10):64552.

25. Waites KB, Chen Y, DeVivo MJ, Canupp KC, Moser SA. Antimicrobial resistance in gram-negative bacteria isolated from the urinary tract in community-residing persons with spinal cord injury. Arch Phys Med Rehabil. 2000;81(6):764-9.

26. Yadav A, Vaidyanathan S, Panigrahi D. Clean intermittent catheterisation for the neuropathic bladder. Paraplegia. 1993;31(6):380-383.

27. [Nosocomial urinary tract infections]. KlimikJ. 2000:19-20.

28. Warren John W. Catheter-associated urinary tract infections. Int J Antimicrob Agents. 2001;17(4):299-303.

29. Dedeic-Ljubovic A, Hukic M. Catheter-related urinary tract infection in patients suffering from spinal cord injuries. Bosn J Basic Med Sci. 2009;9(1):2-9.

30. Maki Dennis. Engineering out the Risk of Infection with Urinary Catheters. Emerg Infect Dis. 2001;7(2):342-347.

31. Wagenlehner FME, Niemetz A, Dalhoff A, Naber KG. Spectrum and antibiotic resistance of uropathogens from hospitalized patients with urinary tract infections: 1994-2000. Int J Antimicrob Agents. 2002;19(6):557-564.
32. Uzun C. . Urinary catheters depending on the risk factors involved in the development of bacteriuria. Edirne; 1995.

33. Andrade MJ, Trepa A, Castro A, Goncalves S. [Urinary tract infections characterization in a spinal cord injury rehabilitation unit]. Acta Med Port. 2009;22(3):215-22.

34. Burns Anthony S, Rivas David A, Ditunno John F. The Management of Neurogenic Bladder and Sexual Dysfunction After Spinal Cord Injury. Spine. 2001;26(Supplement):S129-S136.

35. Stamm Walter E. Catheter-associated urinary tract infections: Epidemiology, pathogenesis, and prevention. Am J Med. 1991;91(3):S65-S71.

36. Falkiner $F$. The insertion and management of indwelling urethral catheters-minimizing the risk of infection. J Hosp Infect. 1993;25(2):79-90.

37. McKinley W, McNamee S, Meade M, Kandra K, Abdul N. Incidence, etiology, and risk factors for fever following acute spinal cord injury. J Spinal Cord Med. 2006;29(5):501-6.

38. Peterson JR, Roth EJ. Fever, bacteriuria, and pyuria in spinal cord injured patients with indwelling urethral catheters. Arch Phys Med Rehabil.1989;70(12):839-41.

39. Perkash I. Long-term urologic management of the patient with spinal cord injury. Urol Clin North Am. 1993;20(3):423-34.

40. Shin JC, Park CI, Kim SH, Yang EJ, Kim EJ, Rha DW. Abdominal ultrasonography findings in patients with spinal cord injury in Korea. J Korean Med Sci. 2006;21(5):927-31. 